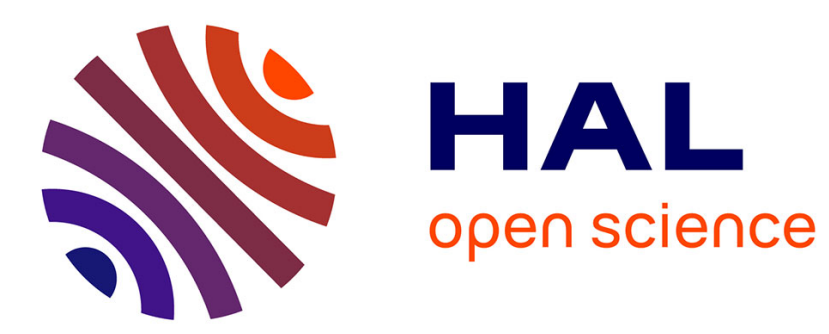

\title{
Evaluation of HD- sEMG grid misalignment with muscle fibers using nonlinear correlation
}

\author{
Amer Zaylaa, Ahmad Diab, Mariam Al Harrach, Sofiane Boudaoud
}

\section{To cite this version:}

Amer Zaylaa, Ahmad Diab, Mariam Al Harrach, Sofiane Boudaoud. Evaluation of HD- sEMG grid misalignment with muscle fibers using nonlinear correlation. International Conference on Advances in Biomedical Engineering (ICABME), Sep 2015, Beirut, Lebanon. 10.1109/ICABME.2015.7323309 . hal-01248864

\section{HAL Id: hal-01248864 \\ https://hal.science/hal-01248864}

Submitted on 29 Dec 2015

HAL is a multi-disciplinary open access archive for the deposit and dissemination of scientific research documents, whether they are published or not. The documents may come from teaching and research institutions in France or abroad, or from public or private research centers.
L'archive ouverte pluridisciplinaire HAL, est destinée au dépôt et à la diffusion de documents scientifiques de niveau recherche, publiés ou non, émanant des établissements d'enseignement et de recherche français ou étrangers, des laboratoires publics ou privés. 


\section{Evaluation of HD- sEMG Grid Misalignment with Muscle Fibers using Nonlinear Correlation}

\author{
Amer Zaylaa, Ahmad Diab \\ AZM center for research in biotechnology \\ Doctoral School for Sciences and Technology \\ Lebanese University (UL), Tripoli, Lebanon \\ amer.zaylaa@hotmail.com, ahmaddiab@ul.edu.lb
}

\author{
Mariam Al Harrach, Sofiane Boudaoud \\ CNRS UMR 7338, BMBI \\ Sorbonne Universités, Université de Technologie de \\ Compiègne (UTC), Labex MS2T, Compiègne, France \\ mariam.al-harrach@utc.fr, sofiane.boudaoud@utc.fr
}

\begin{abstract}
In a recent past, several techniques have been developed to analyze the effect of electrode grid alignment with the muscle fibers. But, they focused on pattern matching of Motor Unit (MU) electrical signatures and not on the interference signal. In this study, we use a method which is widely applied in connectivity and directionality analysis of stochastic and complex signals, namely, the nonlinear correlation coefficient $\left(h^{2}\right)$ on HD-sEMG signal matrices. The approach is first applied on simulated data from recent generation model through an $(8 \times 8)$ simulated electrode matrix, then on real signals using the same grid specifications on the Biceps Brachii. Both simulated and real data were evaluated with three angles of grid alignment with respect to muscle fibers. For this purpose, five parameters were extracted from obtained $\mathrm{h}^{2}$ correlation matrices and tested. According to the obtained results, a relationship between $h^{2}$ values and the electrode matrix alignment seems to exist. However, further efforts are needed to design parameters more sensitive to grid misalignment with respect to muscle fibers.
\end{abstract}

Keywords - Electrode alignment, non linear correlation coefficient, HD-sEMG grid, Biceps Brachii.

\section{INTRODUCTION}

Several factors may affect the variability of surface electromyographic signals (sEMG) such as: the duration and intensity of muscle contraction, the distance of the electrode from the active muscle area, the properties of electrodes, filters, amplifiers and data acquisition system, the quality of contact between the electrodes and the skin in addition to the electrode alignment with muscle fiber $[1,2]$.

Classical recording techniques fall to analyze correctly the muscle activation due to their poor spatial resolution [1]. Recently, multichannel approaches with high number of electrodes were applied [1]. Indeed, the High-Density surface EMG (HD-sEMG) is a non-invasive technique that measures electrical muscle activity with multiple closely spaced electrodes overlying a restricted area of the skin [1]. Unfortunately, such instrumental method is very sensitive to misalignment of the electrode grid according to muscle fibers since all the electrodes should record biased signals.
To guaranty good representativeness of the recorded signals, the longitudinal direction of the grid should be parallel to the muscle fibers in order to align the electrodes to the action potential propagation line [2]. Often, the grid has been simply oriented in line with the longitudinal axis of the muscle belly. For pennated muscles, this becomes more complex [3]. A precise grid alignment with respect to muscle fiber direction is often mandatory for further processing tasks as shape analysis of HD-sEMG data [4], [5] and muscle force estimation [6].

Some automatic realignment approaches have been already proposed on MU fingerprints using HD-sEMG techniques [7] [8]. This induces the study, at low muscle contraction levels, of isolated MUAP topographies. To our knowledge, there is no study that concerns electrode grid alignment using HDsEMG recordings at higher contraction levels and analyzing interference signals. Nonlinear correlation coefficient $\left(h^{2}\right)$ is a widely used approach to evaluate connectivity and directionality analysis of bidimensional complex and stochastic data. It has proven it usefulness in separating preterm uterine sEMG signals from labor ones [9]. We propose to apply this approach, by supposing that grid misalignment with respect to muscle fibers should affect $h^{2}$ values, for assessing misalignment effect on HD-sEMG grid applied on the Biceps Brachii muscle.

In fact, the aim of the proposed study is to find, as a first step, some parameters, estimated from obtained $h^{2}$ correlation matrix and calculated with different grid angles that are sensitive to the electrode grid misalignment with respect to muscle fibers. This parameter will be extracted and tested using simulated and real HD-sEMG data.

For this purpose, we used simulated and real HD-sEMG signals obtained through a 64 electrode grid $(8 \times 8)$ for 3 grid angle values $\left(0,20\right.$ and $\left.40^{\circ}\right)$ during isometric and isotonic contractions of the Biceps Brachii muscle. Thus, five parameters, using nonlinear correlation, are proposed and tested on both simulated and real signals. Finally, the obtained 
results are compared and discussed putting the focus on perspectives of the proposed work.

\section{MATERIALS AND METHODS}

A. Data

1. HD-sEMG model

The model that we used to generate the 64 signals has been described in details in [1, 10]. Briefly, this model has been designed to simulate the signal captured by a single electrode.

TABLE I. Motor Unit (MU) parameters

\begin{tabular}{|c|c|c|c|c|}
\hline $\begin{array}{c}\text { MU } \\
\text { Type }\end{array}$ & $\begin{array}{c}\text { Number } \\
\text { of MU's }\end{array}$ & $\begin{array}{c}\text { MU } \\
\text { diameter } \\
(\mathbf{m m})\end{array}$ & $\begin{array}{c}\text { Number of } \\
\text { fibers/MU }\end{array}$ & $\begin{array}{c}\text { Fiber } \\
\text { diameter } \\
(\boldsymbol{\mu m})\end{array}$ \\
\hline S & 40 & $5 \pm 1$ & $100 \pm 10$ & $45 \pm 5$ \\
\hline FR & 10 & $5.5 \pm 1$ & $150 \pm 15$ & $50 \pm 5$ \\
\hline FIN & 10 & $6 \pm 1$ & $200 \pm 20$ & $55 \pm 5$ \\
\hline FF & 40 & $6.5 \pm 1$ & $250 \pm 25$ & $60 \pm 5$ \\
\hline
\end{tabular}

S(Slow fatigable),FR (Fast Resistant), FIN Fast Intermediate, and $\mathrm{FF}$ (Fast Fatigable) MU types

However, in our study we used it to generate HD-sEMG signals (with a distance of $15 \mathrm{~mm}$ between the left side of the grid and the neuromuscular junction) by applying parallelization techniques and using a high performance computer. This model is composed of different blocks (neural drive, electrical propagation, conductor volume, instrumentation, force) using the formalism of [10]. Model setting values used for constructing our database are reported in Table I concerning the MU repartition. The other parameters are set as in [5] by only adjusting the MU number to 100 . This model represents one biceps muscle composed of 100 MUs disposed in a rectangular territory of $4 \mathrm{~cm}$ in largeness and $2 \mathrm{~cm}$ in depth. Fast MUs are preferentially disposed near the surface and slow ones deeper in the muscle tissue. All the MUs are recruited during simulation. After, the angle effect is simulated by changing the electrode positions of the grid according to the corresponding rotation using polar coordinates. Both simulated and experimental grid are composed of 64 electrodes $(1.6 \mathrm{~mm}$ in diameter and spaced by $4 \mathrm{~mm}$ ) as depicted on Fig.1.

\section{Real Signals}

The method used here is "bivariate" in that we used all the combination between the 64 monopolar channels of a $8 \times 8$ recording electrodes matrix (TMSI REFA 132, Netherland) located on Biceps Brachii. The signals were recorded at BMBI lab. (UTC) on 3 young subjects with 3 measurements during isometric contractions (elbow flexed at $90^{\circ}$ ) at $50 \%$ MVC and using a grid aligned with 0,20 and $40^{\circ}$ according to the longitudinal axis of the muscle. Signals are digitized with a sampling frequency of $2 \mathrm{kHz}$.

\section{B. Method \\ Nonlinear correlation Coefficient $\left(h^{2}\right)$}

The underlying idea is that if the value of $X$ is considered as a function of the value of $Y$, the value of $Y$ given $X$ can be predicted according to a nonlinear regression curve [9, 11]. The nonlinear correlation coefficient between signals $X$ and $Y$ is then calculated as follows (for more details see [12] :

$$
h_{Y \mid X}^{2}=\frac{\sum_{k=1}^{N} Y(k)^{2}-\sum_{k=1}^{N}\left(Y(k)-f\left(X_{i}\right)\right)^{2}}{\sum_{k=1}^{N} Y(k)^{2}}
$$

where $f\left(X_{i}\right)$ is the linear piecewise approximation of the nonlinear regression curve. The estimator $h_{Y \mid X}^{2}$ ranges from 0 ( $Y$ is independent of $X$ ) to 1 ( $Y$ is fully determined by $X$ ). For a nonlinear relationship, $h_{x \mid y}^{2} \neq h^{2}$ and the difference $\Delta h^{2}=$ $h_{x \mid y}^{2}-h_{y \mid x}^{2}$ indicates the degree of asymmetry of the nonlinear coupling.

\section{Parameters}

Five parameters, estimated from $\mathrm{h}^{2}$ correlation matrix, were implemented and extracted after intensive inspection of simulated and real Biceps Brachii signals during a specified maximum isometric isotonic voluntary contraction at $50 \%$ MVC.

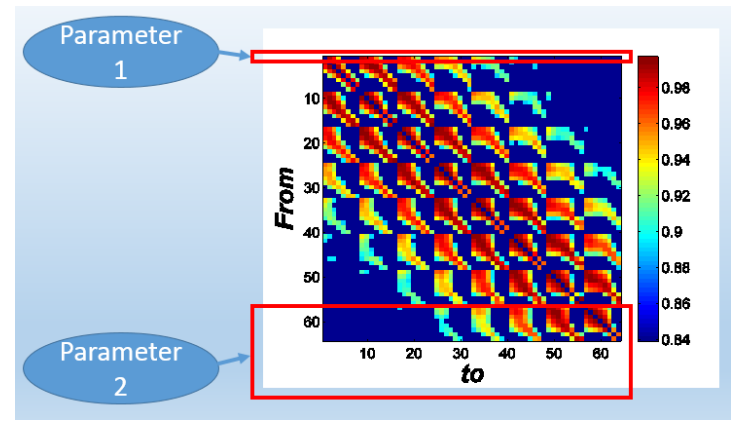

(a)

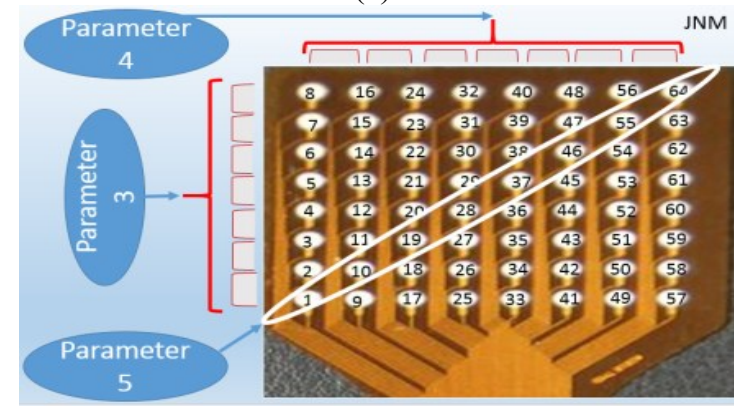

(b)

Fig.1. Parameter description. (a) Parameter 1: $\mathrm{h}^{2}$ mean for first row in correlation matrix, Parameter 2: $\mathrm{h}^{2}$ mean for last 16 rows. (b), Parameter 3: $\mathrm{h}^{2}$ mean for two near electrodes in the 1 st column, Parameter $4: \mathrm{h}^{2}$ mean for two near electrodes in the 1st row, Parameter $5: \mathrm{h}^{2}$ mean for two near electrodes on the diagonal.

In Fig. 1, the $\mathrm{h}^{2}$ mean of the first row in correlation matrix reflects the mean of all electrodes correlation with only the first electrode (parameter 1 in Fig. 1.a). Indeed, it is supposed that this parameter should change according to grid rotation angle. In addition, by visual inspection, we have also noticed variation in the matrix elements with different angles for the last 16 rows defining the parameter 2 (parameter 2 in Fig. 1.a) which represents the mean of all electrodes correlation with the last sixteen electrodes. In addition, the $\mathrm{h}^{2}$ mean of couples of neighbor electrodes in the first column (parameter 3 in Fig. 1.b) represents the parameter 3 . The same process is done on the first row defining the parameter 4 as depicted on Fig. 1.b. 
Finally, we defined the parameter 5 as the $\mathrm{h}^{2}$ mean for the electrodes on the diagonal (parameter 5 in Fig. 1.b) after measuring the nonlinear coefficient between two neighbor electrodes located on this diagonal. This parameter design is motivated by the attempt to express observed differences between $\mathrm{h}^{2}$ matrices at different angles with the higher dynamic allowing an efficient discrimination.

\section{RESULTS}

\section{A. Results on simulated signals}

We try here to test the sensitivity of the implemented parameters with varying grid alignment. So, we calculated them on obtained $\mathrm{h}^{2}$ matrices from simulated HD-sEMG signals generated by the model cited above with grid alignment (rotation according to the grid center) fixed to $0^{\circ}$, $20^{\circ}$ and $40^{\circ}$ respectively.

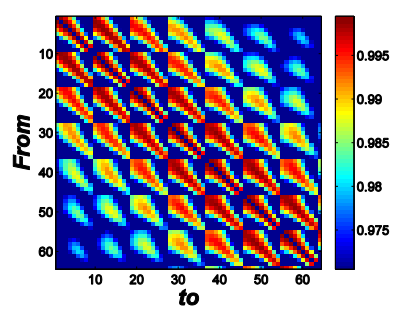

(a)

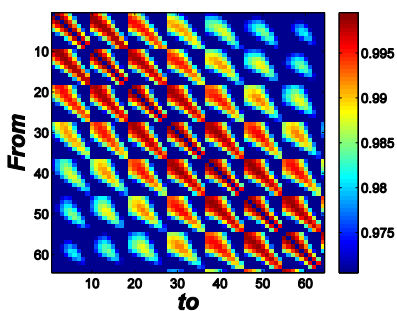

(b)

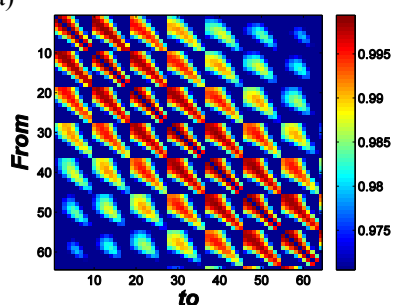

(c)

Fig. 2. $\mathrm{h}^{2}$ nonlinear correlation matrices obtained from simulated HD- sEMG signals at (a) $0^{\circ}$, (b) $20^{\circ}$ and (c) $40^{\circ}$ of grid misalignment (rotation).

Parameters are extracted from $\mathrm{h}^{2}$ correlation matrices depicted on Fig. 2 as colored images for each angle value after thresholding in order to remove the least significant couplings between channels and to increase figure readability. We can observe small differences between the three nonlinear correlation coefficient images. The obtained results for the 5 proposed parameters extracted from $\mathrm{h}^{2}$ correlation matrices are exposed on Fig. 3.

One can observe the poor dynamic of the parameters 3,4 , and 5 that not allows discriminating the three angle values. The parameter 1 and 2 are more sensitive and the obtained trends are monotonically decreasing despite also a small dynamic.

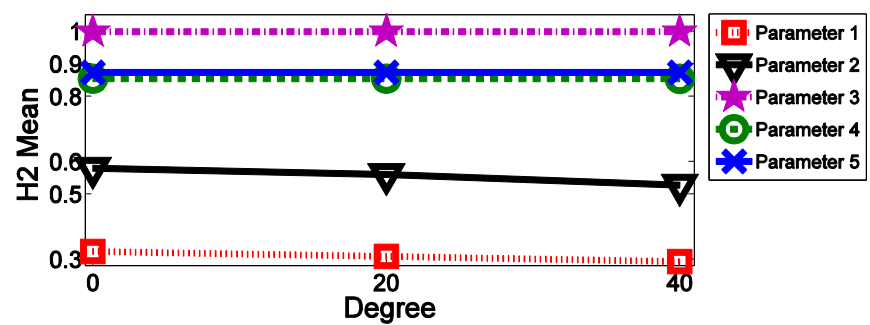

Fig.3. Evolution of $\mathrm{h}^{2}$ mean of all parameters estimated on simulated data in function of grid rotation angle.

\section{B. Results on real signals}

We repeated the same procedure for obtaining the parameters from the $\mathrm{h}^{2}$ correlation matrices of real HD-sEMG Biceps Brachii signals recorded from subjects with the same angle of simulated signals $\left(0^{\circ}, 20^{\circ}\right.$ and $40^{\circ}$ of grid rotation). In Fig. 4, the obtained colored images are represented after nonlinear coefficient thresholding as for the simulated data.

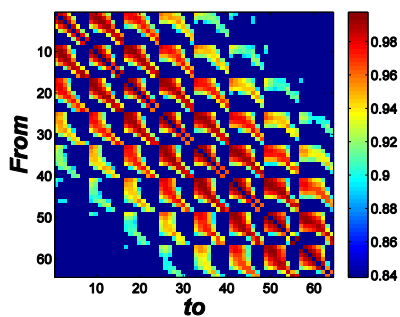

(a)

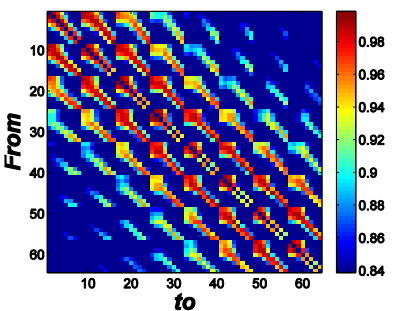

(b)

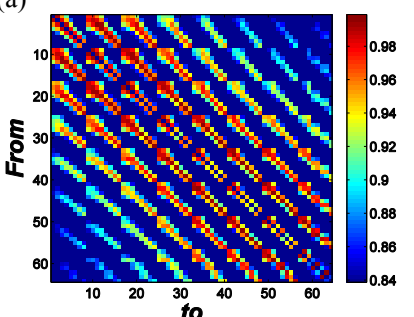

(c)

Fig.4. $\mathrm{h}^{2}$ nonlinear correlation matrix obtained from the Biceps Brachii HDsEMG recorded from subject 1 at (a) 0 degree, (b) 20 degree and (c) 40 degree.

In this figure and in contrary to Fig.2, one can clearly observe modification of the image according to the grid rotation angle. Furthermore, in Fig. 5 are depicted the mean of each parameter according to subject for each angle value. In fact, the parameter 1 decreased monotonically with interesting dynamic as found also in the simulation study. The parameter 4 seems also to be sensitive to the grid alignment but in a non monotonic manner with firstly a net decrease from $0^{\circ}$ to $20^{\circ}$ following by an increase toward $40^{\circ}$. It can be note that this parameter exhibits an important range of variation. Finally, the parameters 2 and 5 exhibit no clear trend and parameter 3 seems to be not sensitive at all to grid angle variation as observed in simulation. However, it is important to note that almost all parameters seem to reach their respective maximal value at $0^{\circ}$ (a grid aligned in the longitudinal direction with respect to the muscle fibers).

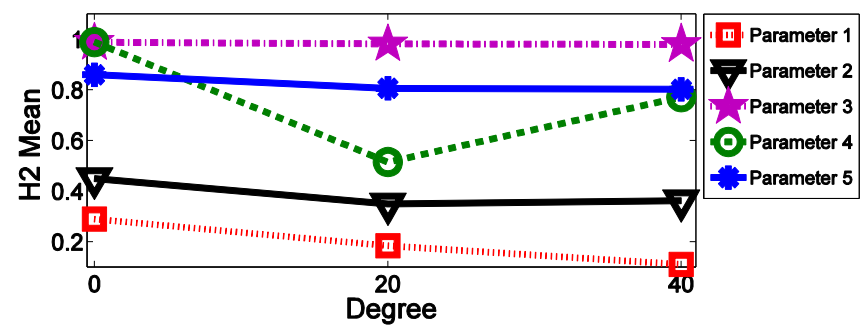

Fig.5. Averaged evolution from the 3 subjects of the 5 parameters estimated on real HD-sEMG signals in function of grid rotation angle. 


\section{DISCUSSION AND CONCLUSIONS}

This exploratory study has the principal objective of evaluating the ability widely known nonlinear method $\left(h^{2}\right)$ to detect possible misalignment of an electrode grid with respect to muscle fibers. Firstly, after recalling the mathematical formalism, the method was tested on simulated HD-sEMG signal matrices, generated by a planar HD-sEMG model. For this purpose, five parameters were constructed, after intensive inspection of the obtained nonlinear correlation coefficient matrices, and tested on these simulated data for three grid rotation angles. It appears that some of these parameters are more sensitive to grid rotation angle than other. This sensitivity seems to be linked to spatial information contained in these matrices. Then, the proposed parameters were experimentally evaluated on HD-sEMG signals obtained from isometric contractions at $50 \% \mathrm{MVC}$ and by varying the grid rotation angle by the same values as for the simulation. On the observed nonlinear correlation coefficient matrices, after visual inspection, we observed an increasingly spreading of connectivity between electrodes when alignment degree increases. This seems to be correct since with increasing grid rotation angle, relationship (dependence) between electrodes that belong to different rows increases. However, this observation is not present on the simulated matrices due to the simulations hypotheses (MU number and volume configuration). Further studies are needed to deeply investigate this hypothesis using experimentation and more realistic modelling. Concerning the behavior of the 5 proposed parameters, we can observe promising monotonic trends (similar to simulation results) with maximum values at $0^{\circ}$ (grid supposed to be well aligned) of some of them according to grid rotation angle. These observations should be confirmed in future works. As perspective, we look forward to applying the methodology described above on a large number of real subjects and with more angles (clockwise and counter clockwise angle variation) to develop an approach that detects the best alignment of the grid according to muscle fibers using nonlinear correlation method.

\section{REFERENCES}

[1] Parker P., Merletti R., "Electromyography: Physiology, Engineering, and Non-Invasive Applications," IEEE Press Series on Biomed. Eng., 2005.

[2] Loeb G.E., C. Gans, "Electromyography for Experimentalists," University of Chicago Press, Chicago, 1986.

[3] Grabiner MD., Koh TJ., Miller GF., "Fatigue rates of vastus medialis oblique and vastus lateralis during static and dynamic knee extension," Journal of orthopaedic research, No. 3, 9: 391-397, 1991.

[4] Ayachi F.S., Boudaoud S., Marque C., "Evaluation of muscle force classification using shape analysis of the $\mathrm{SEMG}$ probability density function: a simulation study,"Medical \& biological engineering \& computing, No. 8, 52: 673-684, 2014.

[5] Al Harrach M., Ayachi F.S., Boudaoud S., Laforet J., Marin F., "Sensitivity evaluation of HOS parameters by data fusion from HD-sEMG grid," in IEEE 2nd International Conference on Advances in Biomedical Engineering (ICABME), Tripoli, Lebanon , 2013.

[6] Allouch S., Al Harrach M., Boudaoud S. , Laforet J., Ayachi F.S., Younes R. , "Muscle Force Estimation Using Data Fusion from High-Density SEMG Grid," in IEEE 2nd International Conference on Advances in Biomedical Engineering (ICABME), Tripoli, Lebanon, 2013.
[7] Grönlund C., Ostlund N., Roeleveld K., Karlsson JS., "Simultaneous estimation of muscle fibre conduction velocity and muscle fibre orientation using 2D multichannel surface electromyogram," Med Biol Eng Comput., 43(1):63-70, 2005.

[8] Gligorijević I., Sleutjes B T H M, De Vos M., Blok J H, Montfoort I. Mijović B., Signoretto M., Van Huffel S., "Motor Unit Tracking Using High Density Surface Electromyograph (HDsEMG)," Automated Correction of Electrode Displacement Errors. Methods of Information in Medicine, 54 (3): 221-6, 2015.

[9] Diab A., Hassan M., Laforêt J., Karlsson B., Marque C., "Estimation of Coupling and Directionality between Signals Applied to Physiological Uterine EMG Model and Real EHG Signals". In XIII Mediterranean Conference on Med and Biol Eng and Comp., Sevilla, Spain, 2013.

[10] Farina D., Merletti R., "A novel approach for precise simulation of the EMG signal detected by surface electrodes," IEEE Trans Biomed Eng, 48:637-46, 2001

[11] Diab A., Hassan M., Boudaoud S., Marque C., Karlsson B., "Nonlinear estimation of coupling and directionality between signals: Application to uterine EMG propagation," IEEE 35th EMBC, 2013.

[12] Pereda E., Quiroga R.Q., Bhattacharya J., "Nonlinear multivariate analysis of neurophysiological signals," Progress in Neurobiology, 77(1-2): p. 1-37, 2005 . 